\title{
A Maximum Information Rate Quaternion Filter for Spacecraft Attitude Estimation
}

\author{
J. Reijneveld*, A. Maas; D. Choukroun \\ Delft University of Technology, Delft, The Netherlands
}

\begin{abstract}
Building on previous works, this paper introduces a novel continuous-time stochastic optimal linear quaternion estimator under the assumptions of rate gyro measurements and of vector observations of the attitude. A quaternion observation model, which observation matrix is rank degenerate, is reduced to a two-dimensional model via a maximum information rate approach. The resulted filter combines the exact treatment of the quaternion process state-dependent noise and the quaternion measurement state-dependent noise under the framework of continuous-time optimal linear filtering. This yields statistically consistent covariance computations within the proposed filter without requiring tuning. The case of white noises in the gyro and vector measurements are considered in this work. This paper also presents the development of a Sun vector determination subsystem for the nanosatelite Delfi-N3xt. Simulations and preliminary experimental validation show that this subsystem, which consists of six four-quadrant Sun sensors, can deliver a Sunspacecraft line of sight with an averaged equivalent angular error of approximately $0.2 \mathrm{deg}$ without the Earth albedo. The performances of the novel filter are illustrated via extensive Monte-Carlo simulations in the case of Delfi-N3xt, where Sun vector measurements, Earth magnetic measurements and gyro measurements are acquired along a $600 \mathrm{~km}$ height Sun synchronous orbit. The proposed filter appears to be insensitive to poor initial conditions and low sampling rates. It converges where a standard extended Kalman filter fails to do so under the same conditions.
\end{abstract}

\section{Introduction}

The attitude quaternion, ${ }^{1} \mathbf{q}$, is the most popular spacecraft attitude parametrization and its mathematical modeling and filtering have been ongoing topics of research for more than forty years. ${ }^{2}$ An important family of quaternion estimators has been developed using the Kalman filtering paradigm. ${ }^{3,4}$ This approach presents known advantages such as statistically meaningful estimates, an easy estimation error covariance analysis, and the straightforward incorporation of parameters other than attitude in the estimation process, to name a few of them. Recently, the well-known quaternion process equation, which features state-multiplicative noises due to gyro noises in the angular velocity measurement, was reformulated in terms of an Itô stochastic differential equation. ${ }^{5}$ This was done in order to comply with the Mean-Square calculus theory underlying the Kalman-Bucy filtering theory. That filter, however, only considered full-quaternion measurements, as can be computed by star-trackers. A related result consisted of the specific Lyapunov differential equation for the quaternion second-order moment. Its driving term, which stemmed from the state-multiplicative quaternion process noise, was explicitly developed and the solution was shown to preserve a trace invariance, which express the quaternion norm invariance in the mean-square sense. Further analysis of the Lyapunov solution showed that it converges, asymptotically, to a scalar matrix [5]. Previously, a $4 \times 4$ q-measurement

\footnotetext{
*Graduate Student, Faculty of Aerospace Engineering, Chair of Space Systems Engineering, Delft, 2629 HS, The Netherlands. J.P.J.Reijneveld@Student.TUDelft.NL

${ }^{\dagger}$ Graduate Student, Faculty of Aerospace Engineering, Chair of Space Systems Engineering, Delft, 2629 HS, The Netherlands. A.S.B.B.Maas@Student.TUDelft.NL

${ }^{\ddagger}$ Asst. Professor, Faculty of Aerospace Engineering, Chair of Space Systems Engineering, Delft, 2629 HS, The Netherlands. D.Choukroun@TUDelft.NL. AIAA, Member.

$\S$ Researcher, Faculty of Aerospace Engineering, Chair of Space Systems Engineering, Delft, 2629 HS, The Netherlands. J.M.Kuiper@TUDelft.NL.
} 
model equation, which was bilinear in $\mathbf{q}$ and in the observation noise, was introduced in [6]. The highlight of that measurement model was that it avoided the undesired linearization procedure but it had the drawback of an increased, four-dimensional, measurement model, where the measurement matrix was rank-degenerated. Some heuristic approaches for measurement model reduction were introduced in [12].

This work builds on the foregoing efforts and introduces a novel stochastic continuous-time quaternion filter using vector observations, as well as an exact approach for reducing the quaternion observation model. Specifically, the contribution of this work is two folds: it combines the exact treatment of the quaternion process state-dependent noise and the quaternion measurement state-dependent noise under the framework of continuous-time optimal linear filtering, and it introduces a maximum information rate optimal linear quaternion estimator from gyro and line-of-sight measurements. For the sake of clarity, the case of white noise errors, both in the gyro and the vector measurements, is tackled first. The more general case, where gyro outputs and vector measurements are corrupted by white noise and randomly drifting biases, is straightforward. Working in continuous-time allows for an easier analysis and development of the filters. Moreover the similarity in the structure of the state-dependent noises yields similar, simple, and exact expressions for the associated intensity matrices. The convergence rate of optimal linear filters hinges essentially on the property of the information rate matrix [7, p.230]. "Richer" information rate matrices accelerate the convergence of the Riccati equation and thus of the estimation process, provided that there is statistical consistency, i.e., that the estimation error statistics is truly expressed by the filter error covariance matrix. The quaternion $4 D$-measurement matrix ${ }^{6}$ being of rank 2 , one can extract 6 possible $2 \times 4$ sub-matrices that have full row rank. By interrogating the 6 possible information rate matrices along the filtering process, the proposed estimator is choosing the path of maximum information rate, within the family of $2 D$-measurement filters. Thus, the amount of information that is lost, when compared to the full $4 D$-measurement filter, is minimized while the computation load of the update stage significantly decreased. The absence of linearization and the exact treatment of the state-dependent noises yield statistically consistent covariance computations within the filter without requiring tuning.

This paper also presents ongoing developments at TU Delft of a Sun vector determination subsystem for the nanosatellite Delfi-N3xt. Physically, this subsystem consists of six four-quadrant Sun sensors located on each of the six panels of the satellite. The proposed algorithm design takes into account geometrical constraints, manufacturing imperfections, and measurement errors at the sensors level, and two approaches are proposed in order to blend the Sun vector measurements into a single spacecraft-Sun line-of-sight measurement.

The performances of the novel reduced quaternion filter are illustrated via extensive Monte-Carlo simulations, using simulated Sun vector measurements, Earth magnetic field measurements, and gyro measurements. In particular, the proposed filter appears to be insensitive to poor initial conditions and low sampling rates and is able to converge where a standard EKF fails to do so under the same conditions.

The paper is organized as follows. Section II is devoted to the development of the reduction approach. Section III presents novel continuous-time optimal quaternion filters. Section IV describes the design of the Sun vector determination subsystem. Section V presents a numerical investigation of the various filters' performances. Finally, conclusions are drawn in Section VI.

\section{Mathematical Modeling}

\section{A. Information Rate in Linear Filtering Theory}

Consider a continuous linear system that is described by the vector (Itô) stochastic differential equation

$$
\mathbf{d x}_{\mathbf{t}}=F(t) \mathbf{x}_{t} d t+G(t) \mathbf{d} \boldsymbol{\beta}_{t}, \quad t \geq t_{0}
$$

and the vector (Itô) observation equation,

$$
\mathbf{d z}_{\mathbf{t}}=H(t) \mathbf{x}_{t} d t+\mathbf{d}_{t}, \quad t \geq t_{0}
$$

The processes $\left\{\boldsymbol{\beta}_{t}, t \geq t_{0}\right\}$ and $\left\{\boldsymbol{\eta}_{t}, t \geq t_{0}\right\}$ are Brownian motion processes with $E\left\{\mathbf{d} \boldsymbol{\beta}_{t} \mathbf{d} \boldsymbol{\beta}_{t}^{T}\right\}=Q(t) d t, Q \geq 0$, $E\left\{\mathbf{d}_{t} \mathbf{d}_{t}^{T}\right\}=R(t) d t, R(t)>0$, independent from each other and from the initial conditions $\mathbf{x}\left(t_{0}\right)$. It is well known that the optimal (minimum variance) filter for the system (1), (2) consists of the following differential 
equations for the conditional mean and covariance matrix:

$$
\begin{aligned}
& \mathbf{d} \widehat{\mathbf{x}}_{t}=F \widehat{\mathbf{x}}_{t} d t+P_{t} H^{T} R^{-1}\left(\mathbf{d z}_{\mathbf{t}}-H \widehat{\mathbf{x}}_{t} d t\right), \\
& \dot{P}_{t}=F P_{t}+P_{t} F^{T}+G Q G^{T}-P_{t} H^{T} R^{-1} H P_{t}, t \geq t_{0}
\end{aligned}
$$

This is the well-known continuous Kalman-Bucy filter, ${ }^{11}$ and the variance equation (4) is its associated Riccati equation. The matrix $H^{T} R^{-1} H$ in (4) is called the information rate matrix. Assuming that the system (1), (2) is uniformly completely observable [7, p. 233] and, thus, that the error covariance matrix $P_{t}$ converges to a unique solution, the information rate matrix conveniently quantifies the rate at which $P_{t}$ converges to that solution. If the observation matrix $H$ is considered a design parameter, then a good design consists in choosing $H$ it such as to maximize the information rate matrix in some sense.

\section{B. Process equation}

It was shown in [5] that the quaternion process equation could be expressed in the framework of Mean-Square Calculus via the following Itô stochastic differential equation:

$$
\begin{aligned}
& \mathbf{d q}_{\mathbf{t}}=F_{I} \mathbf{q}_{t} d t-\frac{1}{2} \Xi\left(\mathbf{q}_{t}\right) \mathbf{d} \boldsymbol{\beta}_{t} ; \quad \mathbf{q}_{t}(0) \stackrel{\text { a.e. }}{=} \mathbf{q}_{0} ; \quad t \in[0, T] \\
& F_{I}=\frac{1}{2}\left(\Omega_{t}-\frac{3 \sigma_{\epsilon}^{2}}{4} I_{4}\right)
\end{aligned}
$$

where $\Omega_{t}$ is a function of the measured angular velocity, $\boldsymbol{\omega}_{t}$, which is corrupted by an additive Brownian motion noise, $\boldsymbol{\beta}_{t}$, such that $E\left\{\boldsymbol{\beta}_{t} \boldsymbol{\beta}_{\tau}^{T}\right\}=\sigma I_{3} \delta(t-\tau)$, and $\mathbf{d} \boldsymbol{\beta}_{t}$ denotes its infinitesimal independent increments. A characteristic of this process equation is its state-multiplicative process noise, where the noise input matrix, $\Xi$, is a known linear matrix function of the quaternion, $\mathbf{q}=\left[\mathbf{e}^{T} q\right]^{T}$, i.e.,

$$
\Xi(\mathbf{q})=\left[\begin{array}{c}
q I_{3}-[\mathbf{e \times}] \\
\mathbf{e}^{T}
\end{array}\right]
$$

As a result, the Lyapunov differential equation governing the time propagation of the second-order moment of $\mathbf{q}_{t}, X_{t}$, was shown to be as follows:

$$
\dot{X}_{t}=F_{I} X_{t}+X_{t} F_{I}^{T}+\frac{\sigma^{2}}{4}\left[\left(\operatorname{tr} X_{t}\right) I_{4}-X_{t}\right] ; \quad X(0)=X_{0}
$$

The damping terms on the main diagonal of $F_{I}$ allows for the process to converge in the mean-square sense (m.s.s.), which ensures, in particular, the invariance of the quaternion norm (m.s.s.), i.e.,

$$
E\left\{\left\|\mathbf{q}_{t}\right\|^{2}\right\}=\operatorname{tr} X_{t}=\operatorname{tr} X_{0} \quad \forall t \geq 0
$$

The unit-norm property of $\mathbf{q}_{t}$ can, thus, be preserved (m.s.s.) by adequately choosing the trace of $X_{0}$ equal to one at the initial time.

\section{Measurement equation}

4D model: Consider the continuous-time equivalent of the four dimensional quaternion measurement model introduced in [6]:

$$
\mathbf{d}_{t}=H(t) \mathbf{q}_{t} d t-\frac{1}{2} \Xi(t) \mathbf{d}_{t}
$$

where the measurement process $\left\{\mathbf{z}_{t}\right\}$ is identically null and the observation matrix $H(t)$ is computed using a single pair of (continuous) vector measurements $\left\{\mathbf{b}_{t}, \mathbf{r}_{t}\right\}$ :

$$
\begin{aligned}
& \mathbf{s}_{t}=\frac{1}{2}\left(\mathbf{b}_{t}+\mathbf{r}_{t}\right) \\
& \mathbf{d}_{t}=\frac{1}{2}\left(\mathbf{b}_{t}-\mathbf{r}_{t}\right) \\
& H(t)=\left[\begin{array}{cc}
-[\mathbf{s} \times] & \mathbf{d}_{t} \\
-\mathbf{d}_{t}^{T} & 0
\end{array}\right]
\end{aligned}
$$


It is assumed that the measurement noise process $\left\{\boldsymbol{\eta}_{t}\right\}$ is a Brownian motion with $E\left\{\boldsymbol{d}_{t} \mathbf{d} \boldsymbol{\eta}_{t}^{T}\right\}=\sigma_{b}^{2} I_{3} d t$, and $\Xi(t)$ denotes the quaternion $4 \times 3$ matrix as defined in (7). Thanks to the linear dependency of the matrix $\Xi(t)$ with respect to $\mathbf{q}_{t}$, an exact expression for the intensity matrix $R(t)$ of the quaternion-dependent noise can be developed (see [6] for the discrete-time case):

$$
R(t)=\frac{\sigma_{b}^{2}}{4}\left[\left(\operatorname{tr} X_{t}\right) I_{4}-X_{t}\right]
$$

It can be easily shown that $R(t)$ is positive definite if $X_{t}$ is positive definite. Henceforth, the information rate matrix $I_{R}(t)$ corresponding to the $4 D$ quaternion measurement model is as follows:

$$
I_{R}(t)=\frac{4}{\sigma_{b}^{2}} H^{T}\left[\left(\operatorname{tr} X_{t}\right) I_{4}-X_{t}\right]^{-1} H
$$

2D model: It was shown in [12] that the four-dimensional H-matrix, which is computed from a single vector observation, is of rank two. This implies that a reduced order two-dimensional measurement model can be equivalently used in a quaternion filter. The measurement update stage in that filter will, thus, have a reduced computational burden. The reduction method described next hinges on the concept of information rate matrix. There are six $2 \times 4$ sub-matrices of $H$, each one of rank two, corresponding to the following pairs of rows: $(1,2),(1,3),(1,4),(2,3),(2,4)$, and $(3,4)$. Their expressions, denoted by $H_{i j}$, are provided in Table 1. Based on the above premisses, a method is suggested next that maximizes the information rate:

Table 1. Six possible reduced quaternion measurement matrices

\begin{tabular}{lccc}
\hline \hline Model & Rows & $H_{i j}$ \\
\hline$R 1$ & $(1,2)$ & {$\left[\begin{array}{cccc}0 & s_{3} & -s_{2} & d_{1} \\
-s_{1} & 0 & s_{1} & d_{2}\end{array}\right]$} \\
$R 2$ & $(1,3)$ & {$\left[\begin{array}{cccc}0 & s_{3} & -s_{2} & d_{1} \\
s_{2} & -s_{1} & 0 & d_{3}\end{array}\right]$} \\
$R 3$ & {$\left[\begin{array}{cccc}0 & s_{3} & -s_{2} & d_{1} \\
-d_{1} & -d_{2} & -d_{3} & 0\end{array}\right]$} \\
$R 4$ & {$\left[\begin{array}{cccc}-s_{3} & 0 & s_{1} & d_{2} \\
s_{2} & -s_{1} & 0 & d_{3}\end{array}\right]$} \\
$R 5$ & {$\left[\begin{array}{cccc}-s_{3} & 0 & s_{1} & d_{2} \\
-d_{1} & -d_{2} & -d_{3} & 0\end{array}\right]$} \\
$R 6$ & {$\left[\begin{array}{cccc}s_{2} & -s_{1} & 0 & d_{3} \\
-d_{1} & -d_{2} & -d_{3} & 0\end{array}\right]$}
\end{tabular}

Step \#1. Extract the six possible $2 \times 4$ reduced measurement matrices, $H_{i j}(t)$ from $H(t)$ (see Table 1$)$. Step \#2. Compute the associated information rate matrices:

$$
I_{R}^{i j}(t)=\frac{4}{\sigma_{b}^{2}} H_{i j}^{T}\left[\left(\operatorname{tr} X_{t}\right) I_{2}-X_{t}^{i j}\right]^{-1} H_{i j}
$$

where $X_{t}{ }^{i j}$ denotes the $2 \times 2$ sub-matrix of $X_{t}$ :

$$
X_{t}^{i j}=\left[\begin{array}{ll}
X(i, i) & X(i, j) \\
X(j, i) & X(j, j)
\end{array}\right]
$$


Step \#3. Choose $(i, j)$ that maximizes the trace of the information rate matrix by solving

$$
\max _{i, j}\left[\alpha_{i j}=\operatorname{tr}\left(I_{R}^{i j}(t)\right)\right]
$$

and use, accordingly, the maximal information rate matrix, $I_{R}^{i j *}(t)$, or equivalently, the observation matrix $H_{i j}^{*}$ in the reduced measurement update stage of the quaternion filter.

\section{Continuous Optimal Linear Quaternion Filters}

In the following are summarized the algorithms of the proposed continuous-time quaternion filters. These filters are exact in the sense that they are the best (minimum variance) linear unbiased estimators of the quaternion given the vector measurement process $\left\{\mathbf{b}_{t}, \mathbf{r}_{t}, t \geq t_{0}\right\}$.

\section{A. 4D model}

$$
\dot{\widehat{\mathbf{q}}}_{t / t}=\left[F_{I}-K H\right] \widehat{\mathbf{q}}_{t / t}, t \geq t_{0}
$$

where

$$
F_{I}(t)=\frac{1}{2}\left(\Omega_{t}-\frac{3 \sigma_{\epsilon}^{2}}{4} I_{4}\right)
$$

and

$$
\begin{aligned}
& \mathbf{s}_{t}=\frac{1}{2}\left(\mathbf{b}_{t}+\mathbf{r}_{t}\right) \\
& \mathbf{d}_{t}=\frac{1}{2}\left(\mathbf{b}_{t}-\mathbf{r}_{t}\right) \\
& H(t)=\left[\begin{array}{cc}
-[\mathbf{s} \times] & \mathbf{d}_{t} \\
-\mathbf{d}_{t}^{T} & 0
\end{array}\right]
\end{aligned}
$$

and the (Kalman) gain matrix $K(t)$ is computed from:

$$
K(t)=\frac{4}{\sigma_{b}^{2}} P_{t / t} H^{T}\left[\left(\operatorname{tr} X_{t}\right) I_{4}-X_{t}\right]^{-1}
$$

The matrices $X_{t}$ and $P_{t / t}$ are computed from the following Lyapunov and Riccati equations, respectively:

$$
\begin{aligned}
& \dot{X}_{t}=F_{I}(t) X_{t}+X_{t} F_{I}^{T}(t)+\frac{\sigma^{2}}{4}\left[\left(\operatorname{tr} X_{t}\right) I_{4}-X_{t}\right], X\left(t_{0}\right) \\
& \dot{P}_{t / t}=F_{I}(t) P_{t / t}+P_{t / t} F_{I}^{T}(t)+\frac{\sigma^{2}}{4}\left[\left(\operatorname{tr} X_{t}\right) I_{4}-X_{t}\right]-\frac{4}{\sigma_{b}^{2}} P_{t / t} H^{T}(t)\left[\left(\operatorname{tr} X_{t}\right) I_{4}-X_{t}\right]^{-1} H(t) P_{t / t}, P\left(t_{0}\right)
\end{aligned}
$$

\section{B. 2D model - Maximum information rate quaternion filter}

The maximum information rate quaternion filter is summarized as follows:

$$
\dot{\widehat{\mathbf{q}}}^{*}{ }_{t / t}=\left[\frac{1}{2}\left(\Omega_{t}-\frac{3 \sigma_{\epsilon}^{2}}{4} I_{4}\right)-K^{*}(t) H_{i j}^{*}(t)\right] \widehat{\mathbf{q}}_{t / t}^{*}, t \geq t_{0}
$$

where $H_{i j}^{*}$ results from Eq. (18) and the (Kalman) gain matrix $K^{*}(t)$ is computed from:

$$
K^{*}(t)=\frac{4}{\sigma_{b}^{2}} P_{t / t}^{*} H_{i j}^{* T}(t)\left[\left(\operatorname{tr} X_{t}\right) I_{2}-X_{t}^{i j}\right]^{-1}
$$




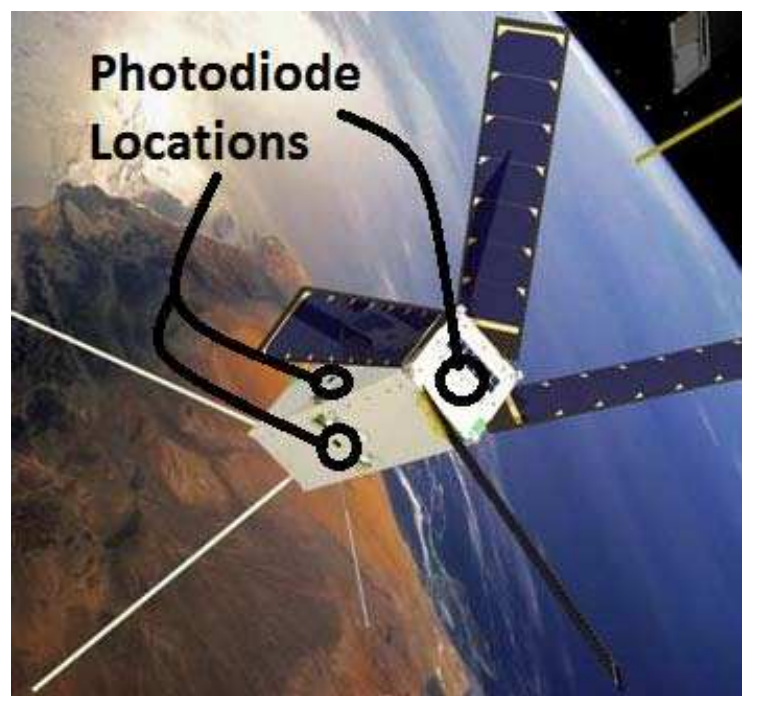

Figure 1. The Delfi-n3Xt satellite with the locations of three of the six Sun sensors. Each sensor is a four-quadrant sensor. The remaining three are in the same locations on the nonvisible sides. (adapted from [13]).

The matrices $X_{t}$ and $P_{t / t}^{*}$ are computed from the following Lyapunov and Riccati equations, respectively:

$$
\begin{aligned}
& \dot{X}_{t}=F_{I}(t) X_{t}+X_{t} F_{I}^{T}(t)+\frac{\sigma^{2}}{4}\left[\left(\operatorname{tr} X_{t}\right) I_{4}-X_{t}\right], X\left(t_{0}\right) \\
& \dot{P}^{*}{ }_{t / t}=F_{I}(t) P_{t / t}^{*}+P_{t / t}^{*} F_{I}^{T}(t)+\frac{\sigma^{2}}{4}\left[\left(\operatorname{tr} X_{t}\right) I_{4}-X_{t}\right]-\frac{4}{\sigma_{b}^{2}} P_{t / t}^{*} H_{i j}^{* T}(t)\left[\left(\operatorname{tr} X_{t}\right) I_{2}-X_{t}^{i j}\right]^{-1} H_{i j}^{*}(t) P_{t / t}^{*}, P\left(t_{0}\right)
\end{aligned}
$$

Remark 1: The state equation is an ordinary differential equation that can be solved using any standard numerical integration scheme. In order to keep the estimated quaternion unitized, and therefore to remain consistent with its physical interpretation as a quaternion of rotation, the estimated quaternion, $\widehat{\mathbf{q}}_{t / t}$ is normalized all along the filtering process. This normalization however is not required by the filtering approach itself, and can be in theory performed outside the filter. Extensive simulations showed that the convergence speed and steady-state accuracy of the estimate were not significantly different between the two techniques.

Remark 2: The above algorithms are independent from the estimate process and the filter covariance computation has been augmented with the Lyapunov equation (29). The computational burden of computing the solution $X_{t}$ can be removed by considering the steady-state solution, $X_{\infty}=\frac{1}{4} I_{4}$ (see [12]). In that case, the Riccati equation (30) becomes:

$$
\dot{P}_{t / t}=F_{I}(t) P_{t / t}+P_{t / t} F_{I}^{T}(t)+\frac{3 \sigma_{\epsilon}^{2}}{16} I_{4}-\frac{16}{3 \sigma_{b}^{2}} P_{t / t} H^{T}(t) H(t) P_{t / t}, P\left(t_{0}\right)
$$

\section{Sun Vector Determination System}

\section{A. Sensor configuration}

The sun vector determination on board Delfi-n3Xt involves a system of six Sun sensors, one on each of the six panels of the CubeSat. Figure 1 depicts the locations of three of these Sun sensors. This configuration was chosen in order to ensure that the Sun line-of-sight will be visible independently of the satellite's attitude. This requirement is critical for mission success, where the aim is to demonstrate three-axis Sun pointing stability. The choice of mounting the Sun sensors on the body, rather than on the solar panels, was dictated by a lack of surface area on the solar panels, which need to be totally employed for power generation. 


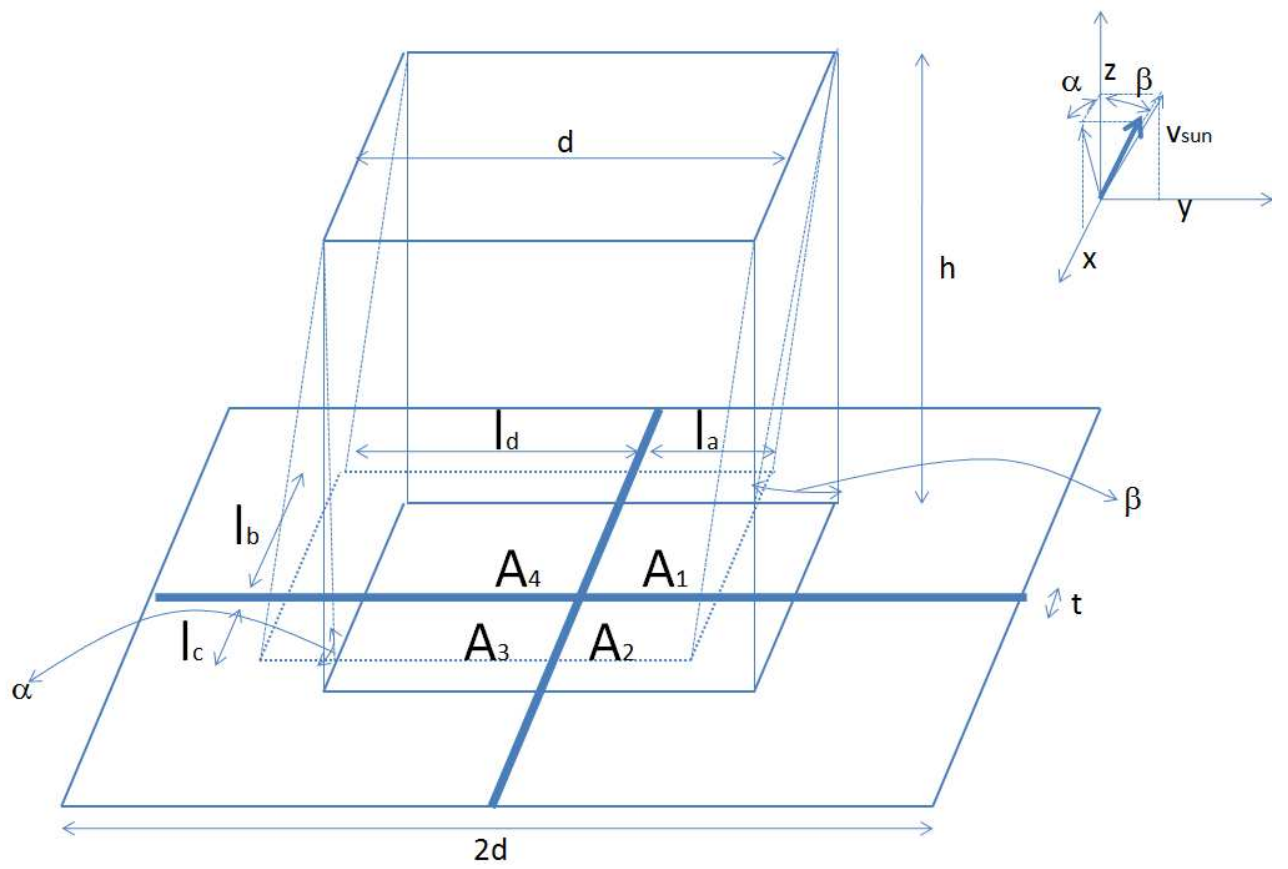

Figure 2. The relation between the lit area on the photodiodes and the direction of the incoming light

\section{B. Sensor Sun vector determination}

\section{Geometric model}

The subsequent analysis focuses on a single Sun sensor. The Sun sensor that is designed is of the quadrant type, where four similar square photodiodes are tightly located next to each together in a square formation. The photodiodes can absorb light, which passes through a square hole that has the same dimensions as each photodiode. Depending on the direction of the incoming light, the area that is lit moves over the four quadrants and a photocurrent is being generated and measured for each of the quadrants. As can be seen from Figure 2, the lit area on each photodiode is direction dependent. This dependency between the sensor orientation and the measured currents stands at the heart of the operation principle of the Sun sensor. It should be already noted that a practical design has to take into account various imperfections aspects, such as geometrical physical constraints, e.g. the angular divergence of the incoming light, geometrical manufacturing constraints, like blunt edges, measurement random noises, as well as perturbations in the signal induced by incoming lights from other sources than the Sun, in particular the Earth (albedo effect). Part of the design developments taking into account these aspects will be presented in the following. We focus next on the ideal case in order to develop the basic Sun vector determination algorithm for a single Sun sensor.

Using Figure 2, we can derive the following relationships

$$
\begin{aligned}
& A_{1}=l_{a} l_{b} \\
& A_{2}=l_{a} l_{c} \\
& A_{3}=l_{c} l_{d} \\
& A_{4}=l_{b} l_{d}
\end{aligned}
$$

where the lengths $l_{a}, l_{b}, l_{c}$, and $l_{d}$ are depending on the direction of the incoming light as follows:

$$
\begin{aligned}
& l_{a}=\left(\frac{d}{2}-\frac{t}{2}-h \tan \beta\right) \\
& l_{b}=\left(\frac{d}{2}-\frac{t}{2}+h \tan \alpha\right) \\
& l_{c}=\left(\frac{d}{2}-\frac{t}{2}-h \tan \alpha\right) \\
& l_{d}=\left(\frac{d}{2}-\frac{t}{2}+h \tan \beta\right)
\end{aligned}
$$


Let $\left\{v_{\text {sun }, i}, i=1,2,3\right\}$ denote the body components of the Sun unit vector. It stems from Figure 2 that these components are related to the angles $\alpha$ and $\beta$ as follows:

$$
\begin{aligned}
\tan \alpha & =\frac{v_{\text {sun }, 1}}{v_{\text {sun }, 3}} \\
\tan \beta & =\frac{v_{\text {sun }, 2}}{v_{\text {sun }, 3}}
\end{aligned}
$$

Furthermore, let $R_{1}$ and $R_{2}$ be two ratios defined as follows

$$
\begin{aligned}
R_{1} & \equiv \frac{A_{1}+A_{2}-A_{3}-A_{4}}{A_{1}+A_{2}+A_{3}+A_{4}} \\
R_{2} & \equiv \frac{A_{3}+A_{2}-A_{1}-A_{4}}{A_{1}+A_{2}+A_{3}+A_{4}}
\end{aligned}
$$

and using (32) to (37) yields

$$
\begin{aligned}
& R_{1}=-\frac{2 h \frac{v_{\text {sun }, 2}}{v_{\text {sun }, 3}}}{(d-t)} \\
& R_{2}=-\frac{2 h \frac{v_{\text {sun }, 1}}{v_{\text {sun }, 3}}}{(d-t)}
\end{aligned}
$$

Since the lit areas and output currents are linearly related, we have found a direct physical relation between the direction of the light, as represented by the Sun vector components $\left\{v_{\text {sun }, i}, i=1,2,3\right\}$, and the output currents of the four quadrants, $\left\{I_{i}, i=1,2,3,4\right\}$ :

$$
\begin{aligned}
& R_{1}=-\frac{2 h \frac{v_{\text {sun }, 2}}{v_{\text {sun }, 3}}}{(d-t)}=\frac{A_{1}+A_{2}-A_{3}-A_{4}}{A_{1}+A_{2}+A_{3}+A_{4}}=\frac{I_{1}+I_{2}-I_{3}-I_{4}}{I_{1}+I_{2}+I_{3}+I_{4}} \\
& R_{2}=-\frac{2 h \frac{v_{\text {sun }, 1}}{v_{\text {sun }, 3}}}{(d-t)}=\frac{A_{3}+A_{2}-A_{1}-A_{4}}{A_{1}+A_{2}+A_{3}+A_{4}}=\frac{I_{3}+I_{2}-I_{1}-I_{4}}{I_{1}+I_{2}+I_{3}+I_{4}}
\end{aligned}
$$

\section{Sun vector determination}

In a physical implementation of the quadrant sensor, the hole will be made in a cover above the sensor, as can be seen in Figure 3. Since the cover will have a certain thickness, this will influence the lengths $l_{a}, l_{b}$, $l_{c}$, and $l_{d}$. The equations giving these length will depend on the incidence angle. From the three beams sketched in Figure 3, it can be seen that:

$$
l=\left\{\begin{array}{cc}
\frac{d}{2}-\frac{t}{2}-h_{1} \tan \theta & \text { if } \theta \leq 0 \\
\frac{d}{2}-\frac{t}{2}-\left(h_{1}+h_{2}\right) \tan \theta & \text { if } 0 \leq \theta \leq \frac{\pi}{2}-\theta_{\text {slope }} \\
\frac{d}{2}-\frac{t}{2}+\frac{t_{\mathrm{cov}}-h_{2}}{\tan \left(\theta_{\text {slope }}\right)}-\left(h_{1}+t_{\mathrm{cov}}\right) \tan \theta & \text { if } \theta \geq \frac{\pi}{2}-\theta_{\text {slope }}
\end{array}\right.
$$

It is possible to derive such equations for $l_{a}, l_{b}, l_{c}$, and $l_{d}$. By using a similar method as in the previous section and (36), this leads to a geometrical model giving a relation for the components of the sun vector as function of $R_{1}$ and $R_{2}$ :

$$
\frac{v_{1}}{v_{3}}=\left\{\begin{array}{cc}
-\frac{R_{2}[d-t+f]+f}{\left(2 h_{1}+t_{\mathrm{cov}}+R_{2} t_{\mathrm{cov}}\right)} & R_{2} \geq-\frac{f\left(h_{2}+2 h_{1}\right)}{h_{2}(f+d-t)-t_{\mathrm{cov}}(d-t)} \\
-\frac{(d-t) R_{2}}{2 h_{1}+h_{2}+R_{2} h_{2}} & 0 \leq R_{2} \leq-\frac{f\left(h_{2}+2 h_{1}\right)}{h_{2}(f+d-t)-t_{\mathrm{cov}}(d-t)} \\
-\frac{(d-t) R_{2}}{2 h_{1}+h_{2}-R_{2} h_{2}} & 0 \geq R_{2} \geq \frac{f\left(h_{2}+2 h_{1}\right)}{h_{2}(f+d-t)-t_{\mathrm{cov}}(d-t)} \\
-\frac{R_{2}[d-t+f]-f}{\left(2 h_{1}+t_{\mathrm{cov}}-R_{2} t_{\mathrm{cov}}\right)} & R_{2} \leq \frac{f\left(h_{2}+2 h_{1}\right)}{h_{2}(f+d-t)-t_{\mathrm{cov}}(d-t)}
\end{array}\right.
$$




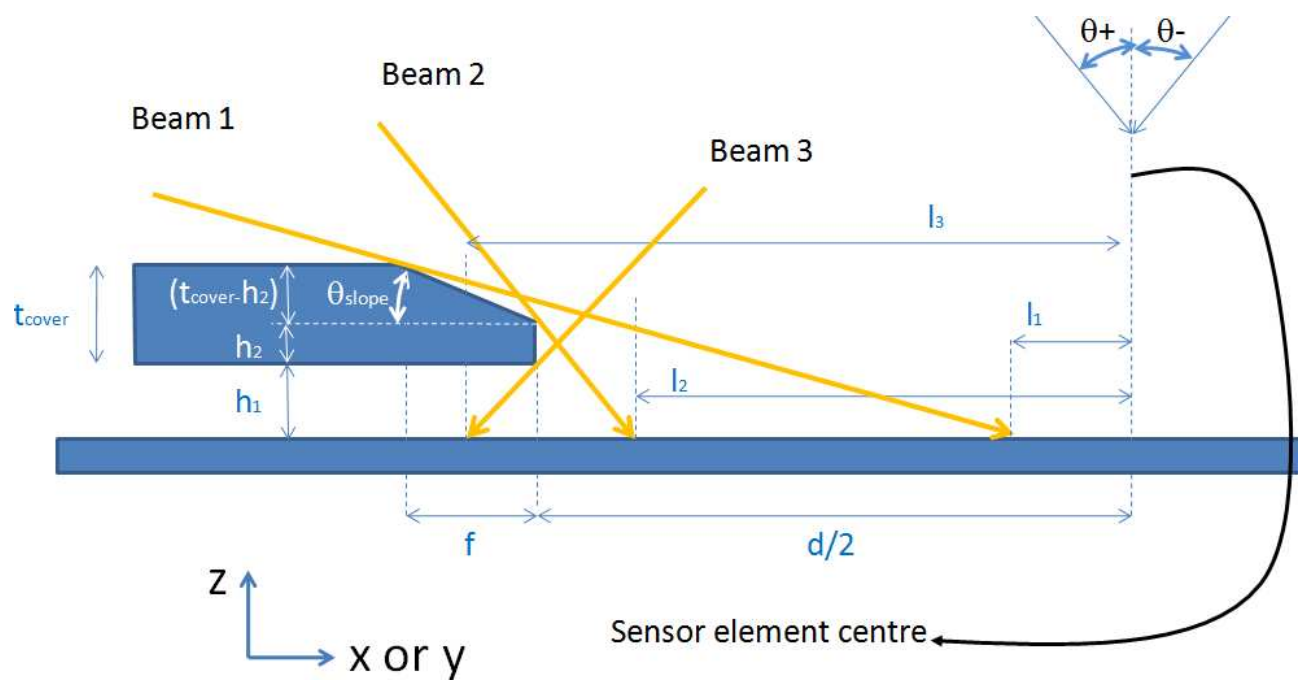

Figure 3. Cross section of the actual sensor. Finite dimensions of the cover are modeled.

Light

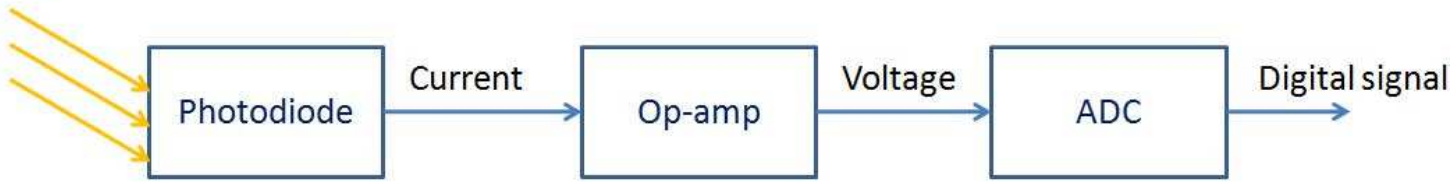

Figure 4. The measurement chain of a single photodiode.

$$
\frac{v_{2}}{v_{3}}=\left\{\begin{array}{cc}
-\frac{R_{1}[d-t+f]+f}{\left(2 h_{1}+t_{\mathrm{cov}}+R_{1} t_{\mathrm{cov}}\right)} & R_{1} \geq-\frac{f\left(h_{2}+2 h_{1}\right)}{h_{2}(f+d-t)-t_{\mathrm{cov}}(d-t)} \\
-\frac{(d-t) R_{1}}{2 h_{1}+h_{2}+R_{1} h_{2}} & 0 \leq R_{1} \leq-\frac{f\left(h_{2}+2 h_{1}\right)}{h_{2}(f+d-t)-t_{\mathrm{cov}}(d-t)} \\
-\frac{(d-t) R_{1}}{2 h_{1}+h_{2}-R_{1} h_{2}} & 0 \geq R_{1} \geq \frac{f\left(h_{2}+2 h_{1}\right)}{h_{2}(f+d-t)-t_{\mathrm{cov}}(d-t)} \\
-\frac{R_{1}[d-t+f]-f}{\left(2 h_{1}+t_{\mathrm{cov}}-R_{1} t_{\mathrm{cov}}\right)} & R_{1} \leq \frac{f\left(h_{2}+2 h_{1}\right)}{h_{2}(f+d-t)-t_{\mathrm{cov}}(d-t)}
\end{array}\right.
$$

We can obtain the third component of the sun vector by using the unit norm constraint.

$$
1=v_{\text {sun }, 1}^{2}+v_{\text {sun }, 2}^{2}+v_{\text {sun }, 3}^{2} \rightarrow \frac{1}{v_{\text {sun }, 3}^{2}}=\frac{v_{\text {sun }, 1}^{2}}{v_{\text {sun }, 3}^{2}}+\frac{v_{\text {sun }, 2}^{2}}{v_{\text {sun }, 3}^{2}}+1 \rightarrow v_{\text {sun }, 3}= \pm \sqrt{\frac{1}{\frac{v_{\text {sun }, 1}^{2}}{v_{\text {sun }, 3}}+\frac{v_{\text {sun }, 2}^{2}}{v_{\text {sun }, 3}^{2}}+1}}
$$

Next, (43) and (44) can be used to obtain the other two normalized components. It is possible to do this for all six sensors, leading to six different estimated sun vectors.

\section{Modeling errors}

In order to identify the influence of all noise source, we first have a look at the measurement chain. The measurement chain has been visualized in Figure 4. Each element in the measurement chain contributes noise to the signal. To see the effects, we will look at each segment individually. The first noise contributor that we consider is the photodiode. Its equivalent circuit has been shown in Figure 5. From the figure, we can find that the current going to the amplifier is:

$$
I_{a m p}=I_{0}-I_{d}-I_{C}-I_{s h}
$$

Since the output of the sensor is slowly varying, we can assume $I_{C}$ to be zero. 


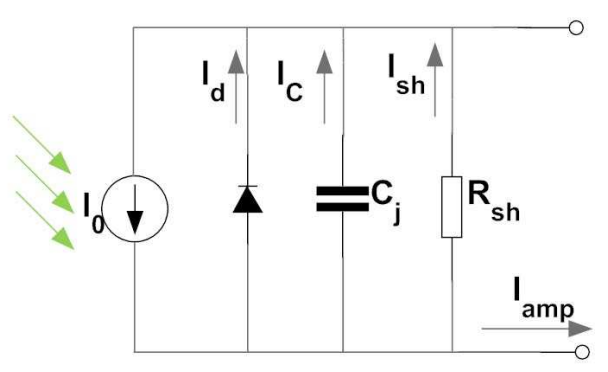

Figure 5. The equivalent circuit of a photodiode. $I_{0}$ is the light generated current, $I_{d}$ is the dark current, $I_{C}$ is the current charging the capacitor, $I_{s h}$ is the current over the shunt resistance, and $I_{a m p}$ is the output current of the photodiode.

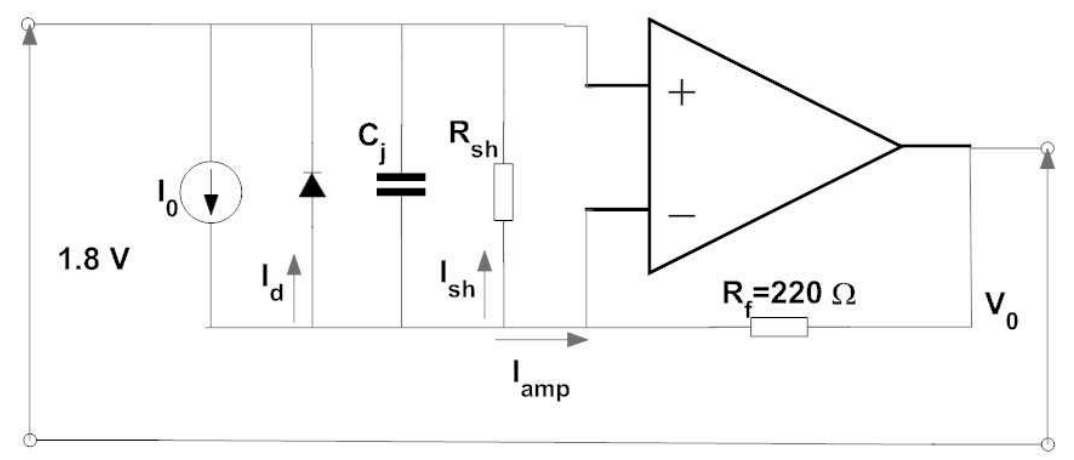

Figure 6. Schematic of the equivalent circuit of the photodiode in combination with the current to voltage converter.

The value of the shunt resistor can be obtained, according to [14], from the voltage-to-current ratio in the vicinity of $0[V]$ and is therefore for this photodiode as follows (see Table 2):

$$
R_{s h}=\frac{10 m V}{I_{D, v=10 m V}}=\frac{10 \cdot 10^{-3}}{67 \cdot 10^{-12}}=1.4925 \cdot 10^{8}[\Omega]
$$

Using Figure 6 , we can now obtain the value for the current through the shunt resistor, $I_{\text {sh }}$, by noting that the current divider is provided as follows:

$$
I_{s h}=\frac{R_{f}}{R_{s h}+R_{f}}\left(I_{0}-I_{d}\right) \approx \frac{R_{f}}{R_{s h}+R_{f}} I_{0}=1.474 \cdot 10^{-6} I_{0}
$$

In the photodiode, there are two sources of noise. These are the thermal noise (or Johnson Noise) of a resistor that approximates the shunt resistance, $R_{s h}$, and the shot noise, resulting from the dark current, the current through the shunt resistor, and the photocurrent. ${ }^{14}$ The noises themselves are: ${ }^{14}$

Table 2. Useful photodiode data taken from datasheet. ${ }^{15}$

\begin{tabular}{lccc}
\hline \hline Parameter & Symbol & Value & Unit \\
\hline \multirow{2}{*}{ Dark current at $10 \mathrm{mV}$} & $I_{d}$ & 67 & {$[\mathrm{pA}]$} \\
Terminal capacitance & $C_{j}$ & 170 & {$[\mathrm{pF}]$} \\
\hline \hline
\end{tabular}




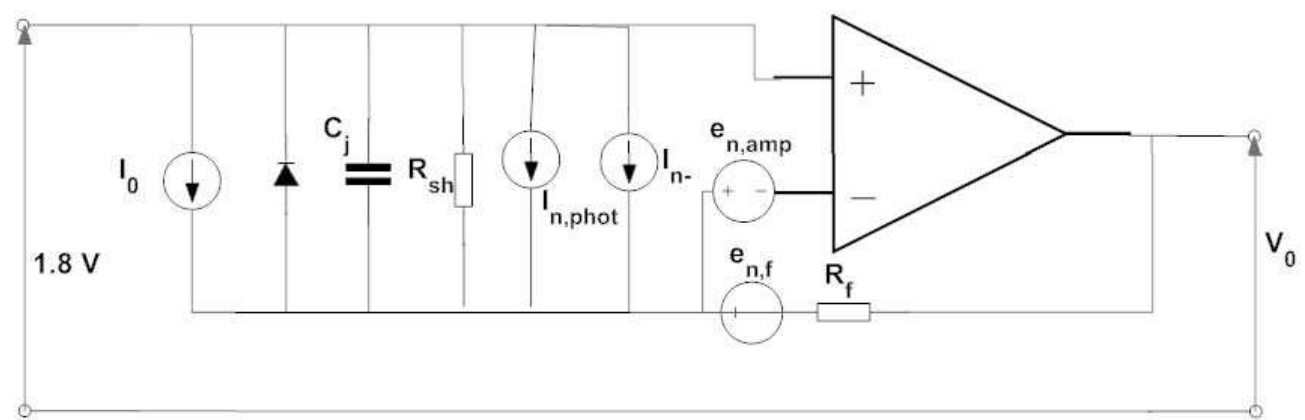

Figure 7. Schematic showing the sources of error in the amplification circuit.

$$
\begin{aligned}
& I_{J}=\sqrt{\frac{4 k T B}{R_{s h}}}=\sqrt{\frac{2 k T}{R_{s h} T_{i}}} \\
& I_{S, d}=\sqrt{2 q I_{d} B}=\sqrt{\frac{q I_{d}}{T_{i}}} \\
& I_{S, s h}=\sqrt{2 q I_{s h} B}=\sqrt{\frac{q I_{s h}}{T_{i}}} \\
& I_{S, 0}=\sqrt{2 q I_{0} B}=\sqrt{\frac{q I_{0}}{T_{i}}}
\end{aligned}
$$

Since

$$
B=\frac{1}{2 T_{i}}
$$

where $B$ is the bandwidth, $k$ is the Bolzmann constant, $q$ is the elementary charge, and $T_{i}$ is the integration time. Furthermore, $I_{J}$ is the root-mean-square (RMS) of the Gaussian distributed Johnson noise, and $I_{S, d}$, $I_{S, s h}$, and $I_{S, 0}$ are the RMS of the Poisson distributed shot noise of the dark current, the current over the shunt resistance and the photocurrent respectively. We can combine these four into a single error source:

$$
I_{n, p h o t}=\sqrt{I_{J}^{2}+I_{S, d}^{2}+I_{S, s h}^{2}+I_{S, 0}^{2}}
$$

For the amplifier circuit with negative feedback, there are three sources of error; a current noise at the $\operatorname{input}\left(I_{n-}\right)$, a voltage noise at the input $\left(e_{n, a m p}\right)$, and a voltage noise source in the feedback circuit due to Johnson noise in the resistor $\left(e_{n, f}\right)$. These noises are visualized in Figure 7. All currents and voltages can be converted to a single voltage at the output of the circuit, $V_{0}$. Using the method that has been described in [16], we can determine the voltage at the output to be:

$$
V_{o}=1.8[V]-R_{f}\left[I_{0}-I_{d}-I_{s h}+I_{n, p h o t}+I_{n-}\right]+\frac{1}{\beta} e_{n, a m p}+\sqrt{\frac{2 k T R_{f}}{T_{i}}}
$$

where $\beta$ is the feedback factor of the circuit (which can be shown ${ }^{16}$ to be $\left[1+\frac{R_{f}}{R_{s h}}\right]$ for frequencies below $4.2[\mathrm{MHz}])$, and $e_{n, a m p}$ and $I_{n-}$ are the voltage and current noise of the op-amp respectively. Also, the datasheet of the selected operational amplifier (op-amp) ${ }^{17}$ shows that the RMS values of the Gaussian distributed noise $e_{n, a m p}$ and $I_{n-}$ are:

$$
\begin{aligned}
& I_{n-}=0.05 \sqrt{\frac{2}{T_{i}}}[\mathrm{pA}] \\
& e_{n, a m p}=10 \sqrt{\frac{2}{T_{i}}}[\mathrm{nV}]
\end{aligned}
$$

The output voltage of the op-amp, $V_{0}$, is connected to the minus port of the analog to digital converter (ADC). The plus port is connected to the $1.8[\mathrm{~V}]$ line, as shown in Figure 8 . This means that the voltage over the ADC input can be written as:

$$
V_{A D C}=1.8[\mathrm{~V}]-V_{o}=R_{f}\left[I_{0}-I_{d}-I_{s h}+I_{n, p h o t}+I_{n-}\right]+\frac{1}{\beta} e_{n, a m p}+\sqrt{\frac{2 k T R_{f}}{T_{i}}}
$$

From [18], it can be found that the quantization noise power is:

\section{1 of 22}




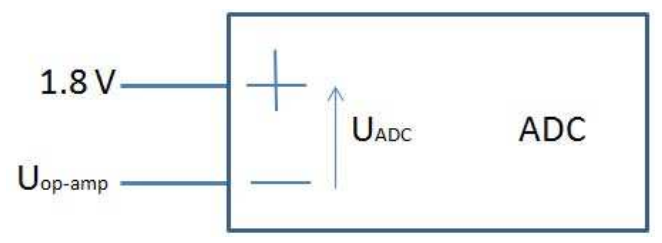

Figure 8. Schematic showing two input ports of the analog to digital converter (ADC).

Table 3. RMS noise of the ADC as function of update rate. ${ }^{19}$

\begin{tabular}{lc}
\hline \hline Update rate $[\mathrm{Hz}]$ & RMS noise $[\mu \mathrm{V}]$ \\
\hline 4.17 & 0.64 \\
8.33 & 1.04 \\
16.7 & 1.55 \\
33.2 & 2.3 \\
62 & 2.95 \\
123 & 4.89 \\
242 & 11.76 \\
470 & 11.33 \\
\hline \hline
\end{tabular}

$$
V_{\text {quantization }, A D C}=\frac{V_{p p}}{\sqrt{12} \cdot 2^{n}}
$$

where $V_{p p}$ is the full-scale input signal voltage, and $\mathrm{n}$ is the number of bits. From the selected electronic design, it is found that $V_{p p}=2.048[V]$. Also, the chosen ADC uses 16 bits to represent the measured value. $^{19}$

Next to the quantization error, the ADC induces also induces another noise. From the datasheet it has been found that depending on the update rate, the ADC adds a voltage noise given by Table 3. The total digital signal will therefore consist of the following contributions:

$$
V_{\text {digital }}=R_{f}\left[I_{0}-I_{d}-I_{s h}+I_{n, p h o t}+I_{n-}\right]+\frac{1}{\beta} e_{n, a m p}+\sqrt{\frac{2 k T R_{f}}{T_{i}}}+V_{n, A D C}
$$

where $V_{n, A D C}$ is the RMS voltage noise of the ADC. From this digital signal, we only consider $R_{f} I_{0}$ to be the signal that we wanted to measure. The rest is considered to be a noise or an error. We can compare the influence of the different errors and noises by comparing their RMS values. An example has been shown in Table 4 for the maximum expected photocurrent $\left(I_{0}=8.2[m A]\right)$ and an update frequency of $470[H z]$. From this analysis it has been seen that the chosen ADC is the limiting factor in the accuracy of the measurement. Since the quantization error is large, we can easily work at the highest update rate of $470 \mathrm{~Hz}$, without it becoming too dominant with respect to the other noise sources. This is required, since the spacecraft will be rotating during the read-out of the quadrants. If too much time is spent reading out a single photodiode, the output of the other sensor has changed, which may lead to incorrect Sun vector measurements. From the mission requirements, it was found that the sun sensor is only operated when the angular velocity has decreased below $10[\% / \mathrm{sec}]$. This means that when reading out a single sensor (consisting of 4 photodiodes), the spacecraft may have rotated an angle of $0.0851^{\circ}$. Since this is much smaller than the required accuracy of the sensor of $3^{\circ}$ and other sources of error (as will be shown later), this effect can be neglected for now.

\section{Spacecraft Sun vector determination}

The algorithm that is used for the Sun vector estimation is still under design. Currently, there are two algorithms under consideration. The first is based on a least square estimate, while the second algorithm looks only at the most trustworthy sensor. For the least square(LSQ) algorithm, two least square estimates 
Table 4. Summary Table for the error sources, types, distributions, and magnitudes, for the Sun sensor measurement chain.

\begin{tabular}{llll} 
Signal & Source & Nature & Magnitude \\
\hline & & & \\
$I_{S}$ & Current due to Sun & Signal & {$[0,12][m A]$} \\
$I_{d i s t}$ & Other Light Sources & Bias & {$[0,4][m A]$} \\
$u_{d}$ & $\varphi$ Dark current & Bias & $15[\mu V]$ \\
$u_{s h}$ & $\varphi$ Leak current & Bias & $3[\mu V]$ \\
$n_{1}$ & $\varphi$ Johnson current noise & Gaussian & $0.2[p A]$ \\
$n_{2}$ & $\varphi$ Signal current shot noise & Poisson & $785[p A]$ \\
$n_{3}$ & $\varphi$ Dark current shot noise & Poisson & $0.1[p A]$ \\
$n_{4}$ & OA equivalent current noise & Gaussian & $1.5[p A]$ \\
$n_{5}$ & OA equivalent voltage noise & Gaussian & $0.3[\mu V]$ \\
$n_{6}$ & OA Johnson voltage noise & Gaussian & $0.03[\mu V]$ \\
$n_{7}$ & ADC quantization noise & Uniform & $9[\mu V]$ \\
$n_{8}$ & ADC noise & Gaussian & $11[\mu V]$ \\
\hline \hline
\end{tabular}

are determined from the output of the six sensors. The cost functions that are minimized to obtain the first and second LSQ estimate, $J_{1}$ and $J_{2}$ respectively, are:

$$
J_{1}=\frac{1}{2} \sum_{\text {allsensors }} \omega_{k}\left|\hat{v}_{\text {Sun }}(a, b)-\tilde{v}_{\text {sun, } \mathrm{k}}\right|^{2}, J_{2}=\frac{1}{2} \sum_{\text {allsensors }} \omega_{k}\left|-\hat{v}_{\text {Sun }}(a, b)-\tilde{v}_{\text {sun }, \mathrm{k}}\right|^{2}
$$

where $\hat{v}_{\text {Sun }}(a, b)$ is the normalized Sun vector:

$$
\hat{v}_{\text {Sun }}(a, b)=\left[\begin{array}{l}
a \sqrt{\frac{1}{a^{2}+b^{2}+1}} \\
b \sqrt{\frac{1}{a^{2}+b^{2}+1}} \\
\sqrt{\frac{1}{a^{2}+b^{2}+1}}
\end{array}\right]
$$

Further, $\omega_{k}$ and $\tilde{v}_{\text {sun,k }}$ are the k-th weight factor and the k-th estimated sun vector respectively. The estimated sun vector is obtained using (44), (43), and (45) and converted from the sensor reference frame to the spacecraft body reference frame. The estimated vectors in the body reference frame are then used in (57). The residuals of the solution to the least square estimate of $J_{1}$ and $J_{2}$ are compared. The $\hat{v}_{\text {Sun }}(a, b)$ that gives the lowest residual is the Sun vector that we are looking for. The least squares problem is solved numerically. The measurements are weighted according to their trustworthiness, i.e. to the size of the signal. Since the signal is dependent on the amount of light that falls on the photodiode, we can use the sum of the currents of the 4 photodiodes as weight factors, i.e.:

$$
\omega_{k}=I_{1, k}+I_{2, k}+I_{3, k}+I_{4, k}
$$

where $I_{i, k}$ is the current of the i-th quadrant of the k-th sensor. As initial condition for the solver, the most trustworthy sun vector measurement is used.

A second algorithm is proposed, which operates via a simple voting scheme: it compares the value of the signals received at the six locations and select the Sun vector produced by the sensor with the maximum sunlight signal(i.e. the sum of the output currents of all four quadrants is highest). This has a very simple implementation, since the estimated Sun vector follows from (44), (43), and (45) directly. 


\section{Simulation results}

A simulator has been build that gives the output currents of all sensors for any Sun location with respect to the satellite. The influence of albedo has been neglected for now. The simulator is capable of taking the Sun's angular divergence into account. It is build on the principle that the spot of the Sun on the sensor element below the hole is a superpositions of an unlimited number of smaller holes that image the sun on the sensor element, as has been shown in Figure 9. This simulator has been used to see the effect of the

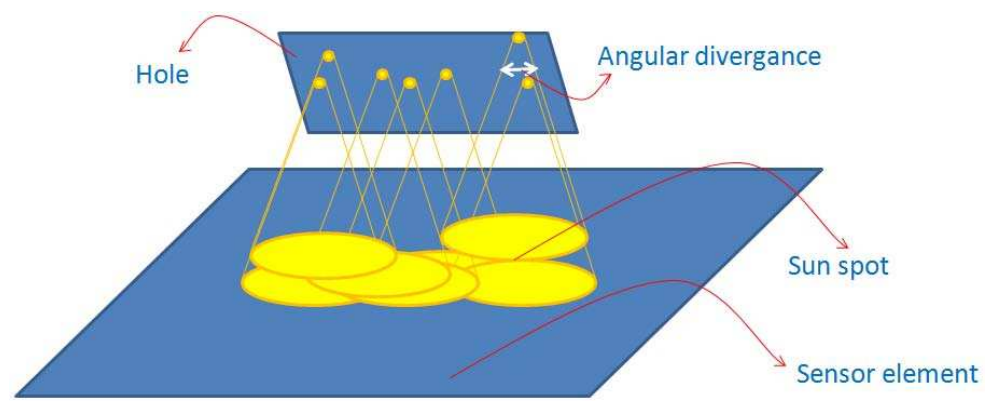

Figure 9. The spot that the Sun leaves on the sensor element through the hole is modeled as a superposition of a large number of small holes that image the Sun.

assumption of zero angular divergence, which was made for the model from Equations (44), (43), and (45), on the sun vector estimate. For different Sun positions with respect to the sensor, the sensor output is determined with the simulator. This output is used to obtain a Sun vector estimate using (44), (43), and (45). The angle difference between the true and the determined Sun vector has been shown in Figure 10. It can be seen that there is a small offset between the actual and the estimated Sun vector. It seems that the model of (44), (43), and (45) is only accurate up to about $0.3^{\circ}$. It should be noted that the spread of the different points are due to the fact that part of the simulator is based on the Monte-Carlo estimation. That is why different runs lead to different simulator outputs and the variation occurs. Next, we do the same, but this time we add the random noises and errors that were found in the previous subsection. The results can be seen in Figure 11. From this figure, it can be seen that the noises and errors have only little influence on the accuracy that can be reached. It is also possible to compare the performance of both the algorithms that were derived. For the weights of (57) we used the total sum of the currents, since this is will lead to a high signal to noise ratio. As a we can see from Figure 12, the highest currents algorithm performs best. It has a lower maximum offset, a lower average, and since it is simpler, a lower computation time. Further research needs to be done to see if more optimal weight factors can be found, which might yield better results for the LSQ algorithm.

\section{E. Experimental results}

A prototype of the Sun sensor has been manufactured. For this prototype, some design parameters are shown in Table 5. The main goals of this prototype were to validate the measurement principle and the derived model. The output current ratio $R_{2}(41)$, of the sensor was simulated in a setup with a beamer as the light source. The results can be found in Figure 13. From this figure, it can be seen that the measurements and model correspond nicely. From this, it can be concluded that the model is an accurate way to describe the behavior of the sensor and that the effect of the beamer's angular divergence on the sensor output is negligible.

\section{Attitude Determination Numerical Example}

Consider a nanosatellite orbiting around the Earth along a circular Sun-synchronous orbit at a height of $600 \mathrm{~km}$. The spacecraft, which experiences free rotational dynamics around its center of mass, has a diagonal body tensor of inertial equal with elements $\left\{J_{x x}, J_{y y}, J_{z z}\right\}=\{0.03699,0.03701,0.00599\}\left[\mathrm{kgm}^{2}\right]$. The spacecraft is assumed to be equipped with three body-mounted rate gyros with a zero-mean Gaussian white noise error of standard deviation $\sigma / \sqrt{\Delta t}=2.310^{-6}[\mathrm{rad} / \mathrm{sec}] . \Delta t$ denotes the time increment between two successive gyro read-out and is chosen equal to 0.25 second. The spacecraft is also equipped with a triad 


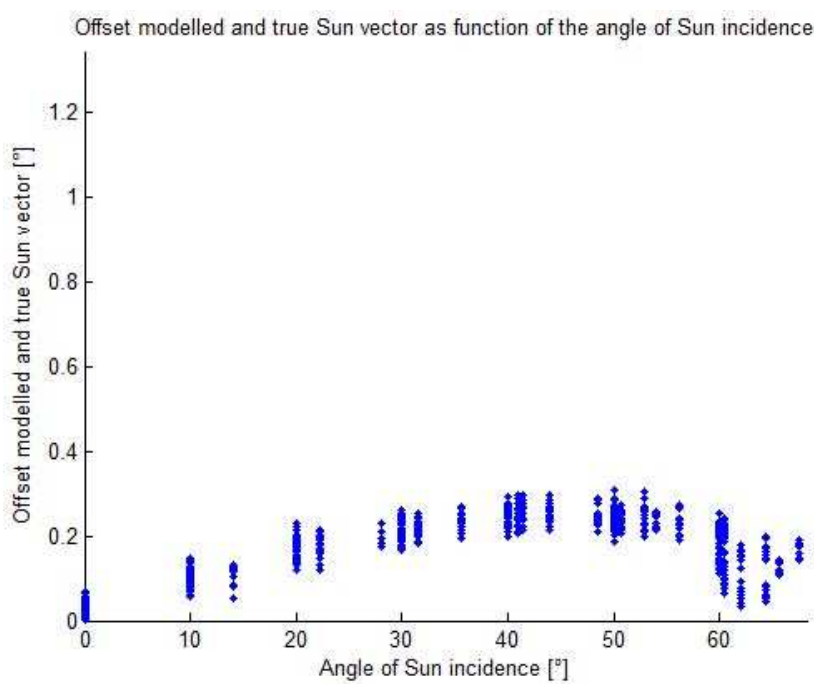

Figure 10. Offset between the actual Sun vector and that derived from the model(Equations 44, 43, and 45) as function of the angle between the Sun and sensor normal. Noises and errors have not been included in these simulations.

Table 5. Design parameters of the manufactured prototype. The symbols have been explained in Figure 3.

\begin{tabular}{lcc}
\hline \hline Parameter & Value & Unit \\
\hline$d$ & 5 & {$[\mathrm{~mm}]$} \\
$h_{1}$ & 1 & {$[\mathrm{~mm}]$} \\
$h_{2}$ & 0 & {$[\mathrm{~mm}]$} \\
$\theta_{\text {slope }}$ & 30 & {$\left[{ }^{\circ}\right]$} \\
$t_{\text {Cover }}$ & 1.1 & {$[\mathrm{~mm}]$} \\
\hline \hline
\end{tabular}

of magnetometers (MGM), which measurements are corrupted with zero-mean Gaussian white noises, and the Sun sensors suite described in the previous section The MGM equivalent angular standard deviation, $\sigma_{b} / \sqrt{\Delta t_{M G M}}$, is equal to 1 degree, which is typical for coarse magnetic field measurements. In order to test the filtering algorithm in computationally constrained conditions, it was assumed that the MGM and Sun sensor sampling frequencies were 20 times slower than that of the gyros. The simulated Earth magnetic field corresponds to the IGRF11 model. The inertial coordinates frame corresponds to the local NorthEast-Nadir frame existing at the initial time, which is assumed to be the local ascending node time epoch. The simulations runs last 12000 seconds which corresponds to approximately two orbital revolutions of the satellite around the Earth. The simulations results are summarized next.

Figure 14 depicts the time histories of $\sqrt{\operatorname{tr} P_{t / t}}$, the square-root of the trace of the error covariance matrix, for the various filters. All filters started with the same initial conditions for $P$ as well as for $X$, i.e, $P(0)=X(0)=5 I_{7}$. The black line and the blue line correspond to the $4 D$-measurement filter (full filter) and to the $2 D$-maximum information rate filter (RMAX filter), respectively. Each one of the other lines correspond to one of the possible $2 D$-measurement quaternion filters. For instance, the R1 filter implements at each update the sub-matrix obtained by extracting the rows 1 and 4 of the $4 D$ observation matrix (See Table 1). The relative efficiency of the RMAX filter, within the family of the $2 D$-measurement filters, clearly appears from the plot. It is interesting to notice that the RMAX filter does not need to compute an inherent measurement matrix, but merely picks the appropriate one from a limited number of possible values, as a result of the information rate maximization, and uses it accordingly in the update stage. By doing so, it differentiates itself from the start, and increases its gap whenever each of the possible model has got a better observability. As can be seen from Fig. 14, the full filter (blue) converges quicker than any of the 


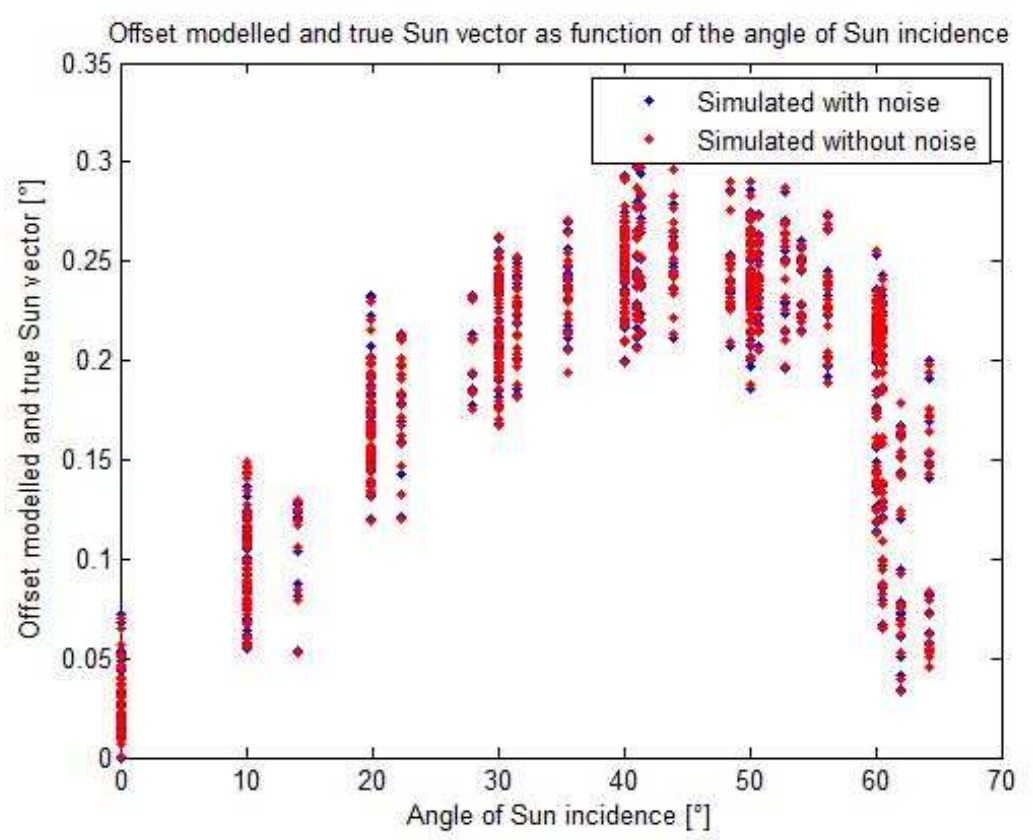

Figure 11. Offset between the actual Sun vector and that derived from the model(Equations 44, 43, and 45) as function of the angle between the Sun and sensor normal. Noises and errors have been included in the blue simulations.

2D-measurement filters, including the RMAX filter, and remains significantly lower asymptotically. This is no surprise since the information rate of that filter beneficiates from a "richer" $(4 D)$ observation matrix, i.e., its Frobenius norm is approximately twice that of any of its six $2 \times 4$ sub-matrices. This, therefore, yields a steeper convergence rate.

In addition to the free-dynamics (tumbling) scenario with an initial angular velocity of $10[\mathrm{deg} / \mathrm{sec}]$ around the three axis, a pure-spin scenario was considered were the $\mathrm{S} / \mathrm{C}$ was simulated spinning at the rate of $\sin (2 \pi / 150)[\mathrm{deg} / \mathrm{sec}]$ around its axis of lowest inertia that is aligned with an arbitrary inertial direction. The sampling times of the MGM and the Sun sensors were reduced to 2.5 second. For the sake of comparison, an additive extended Kalman filter (EKF) was implemented under the same conditions and with the same statistical noise assumptions. A brief review of the EKF is provided in the Appendix for completeness. Figure 15 shows the performances of the filters, on a single run, in terms of the quaternion error of rotation. That quaternion is computed via quaternion multiplication between the true and the normalized estimated quaternion. Its fourth component yields the angular estimation error, $\delta \phi$, and the first three components form the vector part, $\delta q(1), \delta q(2), \delta q(3)$. The three filters show similar performances in the spinning $\mathrm{S} / \mathrm{C}$ case. There, the angular error converges to a steady-state level of 0.1 degree, that is one tenth of the noise level present in the Earth magnetic field measurements. On the other hand, in the tumbling S/C case, the EKF fails to converge. This likely stems from its known sensitivity to initial conditions and modeling errors. The plots of the full and the RMAX filters show a slight advantage to the full filter. This result is supported by the analysis of the filters, previously described. The information rate is greater in the full filter than in the RMAX filter, which leads to a quicker convergence of the errors' second and first order moments of the full filter. In steady-state, both filters reach similar levels of accuracy to those of the spinning S/C case.

An alternative thus appears in the design of the quaternion filter where speed of convergence can be traded for computational burden (induced by the $4 D$-measurement filter). Notice that another nice feature of the RMAX filter is a better numerically conditioned algorithm since $H_{i j}(t)$ is a full row-rank matrix.

In order to investigate the statistical consistency of the proposed maximal information rate filter, RMAX, the scenario of the tumbling S/C was simulated. The sampling rates of the gyro, the MGM, and the Sun sensors were chosen to be $10 \mathrm{~Hz}$. This was done in order to approach the continuous-time behavior of the filter. These sampling rates are realistic. A set of 60 Monte-Carlo simulations was run, with time-spans limited to 1000 seconds since convergence was reached far before. Figure 16 features the plots of the Monte- 


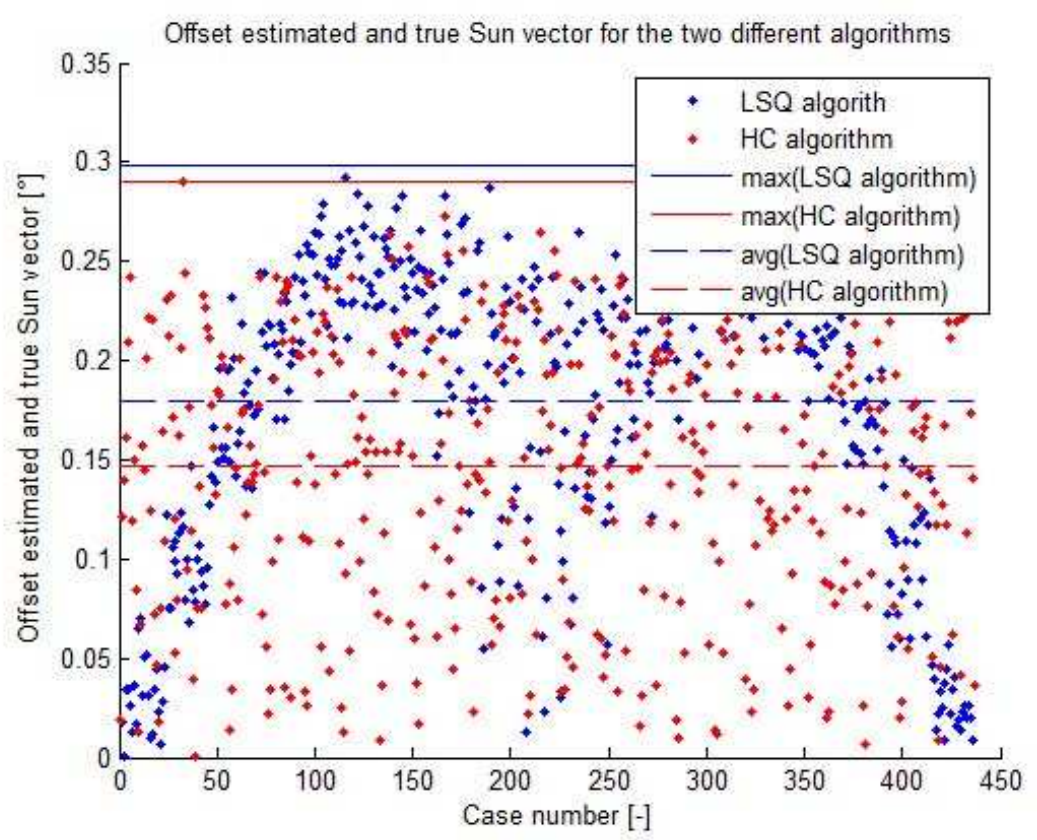

Figure 12. Offset between the actual Sun vector and that estimated using the least squares algorithm (LSQ algorithm) and the highest currents algorithm (HC algorithm). The offset has been evaluated for all possible Sun locations with 1 azimuth and elevation step size. The average and maximum values of the algorithms have also been shown.

Carlo (MC) standard deviations of the quaternion-error vector part (dashed line) and of the corresponding (square-root) element in the $P_{t / t}$ matrix (solid line). The two plots are close to each other which illustrates the statistical consistency of the proposed maximal information rate filter.

\section{Conclusion}

In this work, exploiting the rank degeneracy of the quaternion $4 D$-measurement matrix, a novel approach was proposed, based on the information rate matrix, for developing a $2 D$-measurement quaternion filter. This filter handles without approximation the state-dependent noises appearing both in the process and in the measurement models. By continuously probing the norm of the information rate matrix, within the family of possible reduced measurement matrices, it can switch the model on-line, which ensures the greatest instantaneous information rate. This results in a decrease in the computation burden and a better numerical conditioning. This work also presented the developments of a Sun vector determination subsystem for the triple cubesat Delfi-N3xt. The performances of the novel filter were tested using simulated Sun vector measurements, Earth magnetic field measurements, and gyro measurements. Its performances compared favorably with respect to the full quaternion filter. It proved to perform satisfactorily in spite of quick dynamics, poor initial conditions, and low sampling rates, which caused a standard additive quaternion EKF to fail. Extensive Monte-Carlo simulations illustrated the statistical consistency of the proposed estimator, which is achieved without requiring additional tuning.

\section{Appendix A. Review of the Quaternion Additive Extended Kalman Filter}

The goal of this section is to briefly review the typical measurement update stage of the AEKF. For convenience we rewrite the non-linear vector measurement equation

$$
\mathbf{b}_{t}=A\left(\mathbf{q}_{t}\right) \mathbf{r}_{t}+\boldsymbol{\delta} \boldsymbol{b}
$$

where the attitude matrix denoted by $A$ is a known function of $\mathbf{q}_{t}$. Denoting by $\mathbf{h}\left(\mathbf{q}_{t}\right)$ the non-linear measurement function, $A\left(\mathbf{q}_{t}\right) \mathbf{r}_{t}$, then the $3 \times 4$ linearized measurement matrix, when evaluated at $\widehat{\mathbf{q}}_{t / t}$, is 


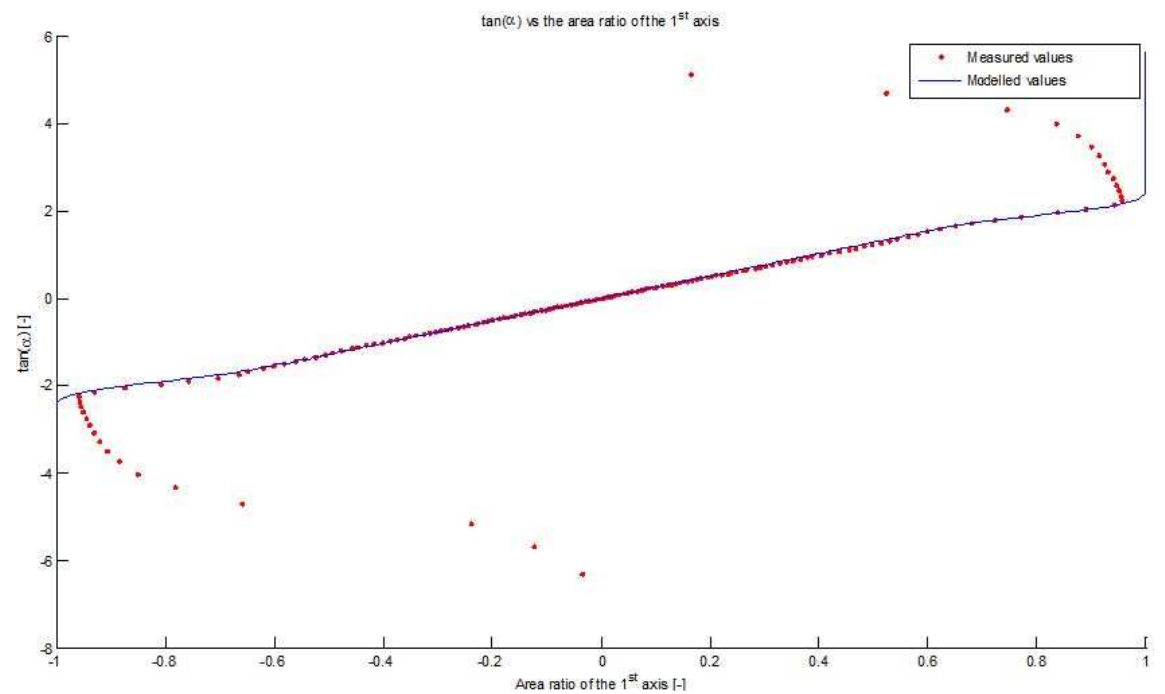

Figure 13. The results of measurement of $R_{2}$ as function of the light incidence angle $\alpha$. The modeled output using Equation 44 has been shown as well.

expressed as follows,

$$
\begin{aligned}
\bar{H}\left(\widehat{\mathbf{q}}_{t / t}\right) & =\nabla_{\mathbf{q}} \mathbf{h}\left(\widehat{\mathbf{q}}_{t / t}\right) \\
& =2\left[\widehat{\mathbf{e}}^{T} \mathbf{r}_{t} I_{3}+\widehat{\mathbf{e}} \mathbf{r}_{t}^{T}-\mathbf{r}_{t} \widehat{\mathbf{e}}^{T}+2 \widehat{q}[\mathbf{r} \times], \quad \widehat{q} \mathbf{r}_{t}+[\mathbf{r} \times] \widehat{\mathbf{e}}\right]
\end{aligned}
$$

where $\mathbf{e}$ and $q$ are the vector and scalar part of $\mathbf{q}_{t}$, respectively, and where the time indices were omitted for the sake of notational simplicity. The AEKF algorithm is then formulated as follows:

$$
\dot{\hat{\mathbf{q}}}_{t / t}=F_{I}(t) \widehat{\mathbf{q}}_{t / t}+\bar{K}(t)\left(\mathbf{b}_{t}-\mathbf{h}\left(\widehat{\mathbf{q}}_{t / t}\right)\right)
$$

where

$$
\begin{aligned}
& \bar{K}(t)=\frac{1}{\sigma_{b}^{2}} P_{t / t} \bar{H}\left(\widehat{\mathbf{q}}_{t / t}\right)^{T} \\
& \dot{P}_{t / t}=F_{I}(t) P_{t / t}+P_{t / t} F_{I}^{T}(t)+\frac{\sigma^{2}}{4} \Xi\left(\widehat{\mathbf{q}}_{t / t}\right) \Xi^{T}\left(\widehat{\mathbf{q}}_{t / t}\right)-\frac{1}{\sigma_{b}^{2}} P_{t / t} \bar{H}^{T}\left(\widehat{\mathbf{q}}_{t / t}\right) \bar{H}\left(\widehat{\mathbf{q}}_{t / t}\right) P_{t / t}
\end{aligned}
$$

Notice that the estimation errors, which are present in the matrices $\Xi\left(\widehat{\mathbf{q}}_{t / t}\right)$ and $\bar{H}\left(\widehat{\mathbf{q}}_{t / t}\right)$ induce a sensitivity of the covariance computation to the initial estimate conditions. This is typical to extended Kalman filters. Notice that this feature is avoided in the proposed filters.

\section{Acknowledgements}

This research was partly supported by THE ISRAEL SCIENCE FOUNDATION (grant No. 1546/08) and by the Delfi Satellite Program of TUD - Delft University of Technology

\section{References}

${ }^{1}$ Wertz, J.R. (ed.), Spacecraft Attitude Determination and Control, D. Reidel, Dordrecht, The Netherlands, 1984.

${ }^{2}$ Crassidis, J., Markley, F.L., and Cheng, Y., "Nonlinear Attitude Filtering Methods ," Journal of Guidance, Control, and Dynamics, Vol. 30, No. 1, 2007, pp. 12-28.

${ }^{3}$ Lefferts, E.J., Markley, F.L., and Shuster, M.D., "Kalman Filtering for Spacecraft Attitude Estimation," Journal of Guidance, Control, and Dynamics, Vol. 5, Sept.-Oct. 1982, pp. 417-429.

${ }^{4}$ Bar-Itzhack, I.Y., and Oshman, Y., "Attitude Determination from Vector Observations: Quaternion Estimation," IEEE Transactions on Aerospace and Electronic Systems, Vol-AES-21, Jan. 1985, pp. 128-136. 
${ }^{5}$ Choukroun, D., "Novel Quaternion Stochastic Modelling and Filtering," AIAA Guidance, Navigation and Control Conference, AIAA-2008-6299, Honolulu, Hawai, Aug. 18-21, 2008, and Journal of Astronautical Sciences, accepted for publication.

${ }^{6}$ Choukroun, D., Oshman, Y., and Bar-Itzhack, I.Y., "Novel Quaternion Kalman Filter," IEEE Transactions on Aerospace and Electronic Systems, Vol. AC-42, No. 1, Jan. 2006, pp. 174-190.

${ }^{7}$ Jazwinski, A.H., Stochastic Processes and Filtering Theory, New York: Academic, 1970.

${ }^{8}$ Choukroun, D., Novel Methods for Attitude Determination Using Vector Observations, Ph.D. Thesis, Faculty of Aerospace Engineering, Technion-Israel Inst. of Techn., May 2003, pp. 283-284.

${ }^{9}$ Bellman, R., Introduction to Matrix Analysis, McGraw-Hill, New-York, 1960.

${ }^{10}$ Mendel, J.M., Lessons in Estimation Theory for Signal Processing, Communication, and Control, Prentice-Hall PTR, Englewood Cliffs, New Jersey, 07632.

${ }^{11}$ Kalman, R.E., and Bucy, R.S., "New Results in Linear Filtering and Prediction Theory," Trans. ASME, J. Basic Eng., Ser. D, Vol. 83, March 1961, pp. 95-108.

${ }^{12}$ Choukroun, D., "Novel Results on Quaternion Modelling and Estimation from Vector Observations," AIAA Guidance, Navigation and Control Conference, AIAA-2009-6315, Chicago, Illinois, Aug. 10-13, 2009.

${ }^{13}$ TU Delft, Delf - a nanosatellite development line, http://www.delfispace.nl/.

${ }^{14}$ Hamamatsu, Photodiode Technical Information, http://sales.hamamatsu.com/assets/ applications/SSD/photodiode_technical_information.pdf.

${ }^{15}$ Hamamatsu, Si PIN photodiode S5981, http://sales.hamamatsu.com/assets/pdf /parts_S/s5980_etc_kpin1012e04.pdf

${ }^{16}$ Burr-Brown, Noise Analysis Of FET Transimpedance Amplifiers, http://focus.ti.com/lit/an/sboa060/sboa060.pdf

${ }^{17}$ Analog Devices, AD8615/AD8616/AD8618, $\quad$ http://www.analog.com/static/importedfiles/data_sheets/AD8615_8616_8618.pdf

${ }^{18}$ Wertz, J.R. (e.d.), Space Mission Analysis And Design, Kluwer Academic Publishers, September 1999.

${ }^{19}$ Analog Devices, AD7794/AD7795, http://datasheet.octopart.com/AD7795BRUZ-Analog-Devices- datasheet5401700.pdf 


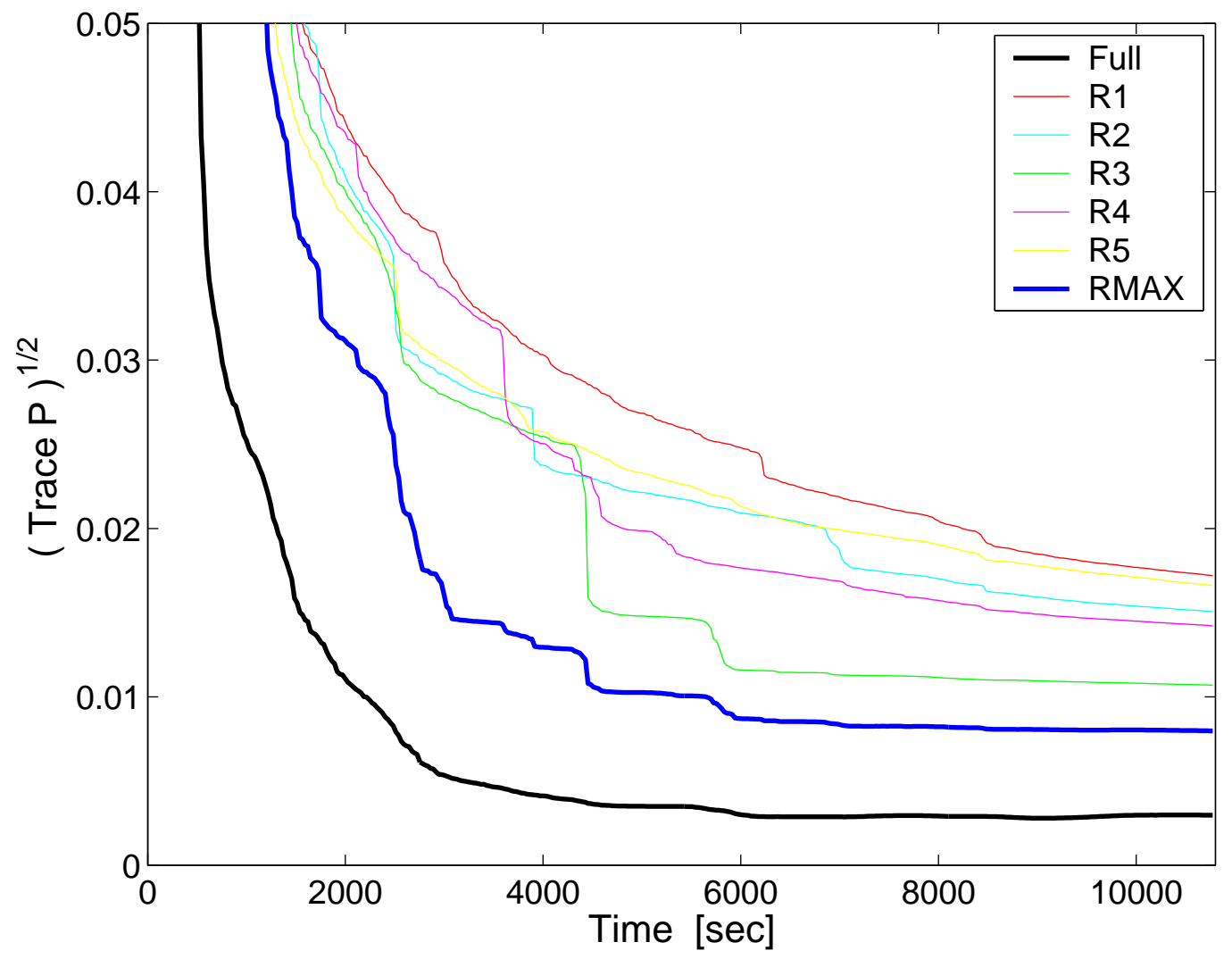

Figure 14. Time histories of $\sqrt{\operatorname{tr} P_{t / t}}$ for the various filters. The blue line shows that the information rate is maximized within the family of 2-D measurement quaternion filters. Single run. 


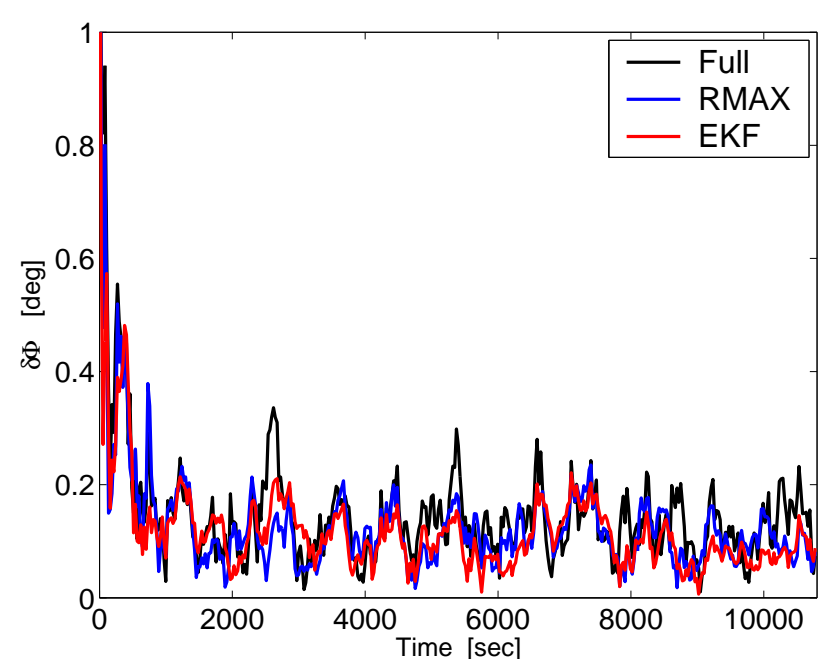

(a) Spinning S/C: Angular estimation error.
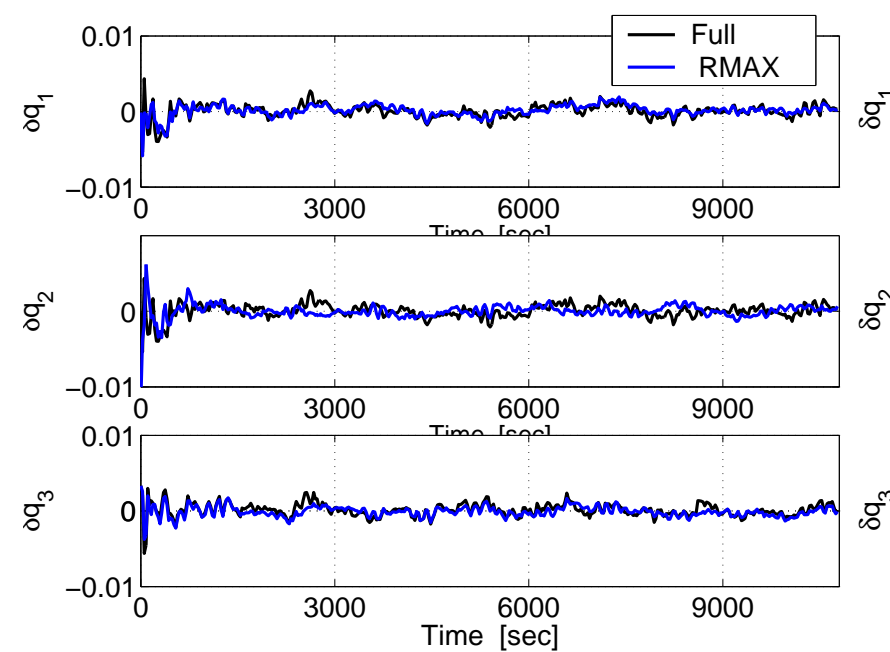

(c) Spinning S/C: Quaternion error vector part.

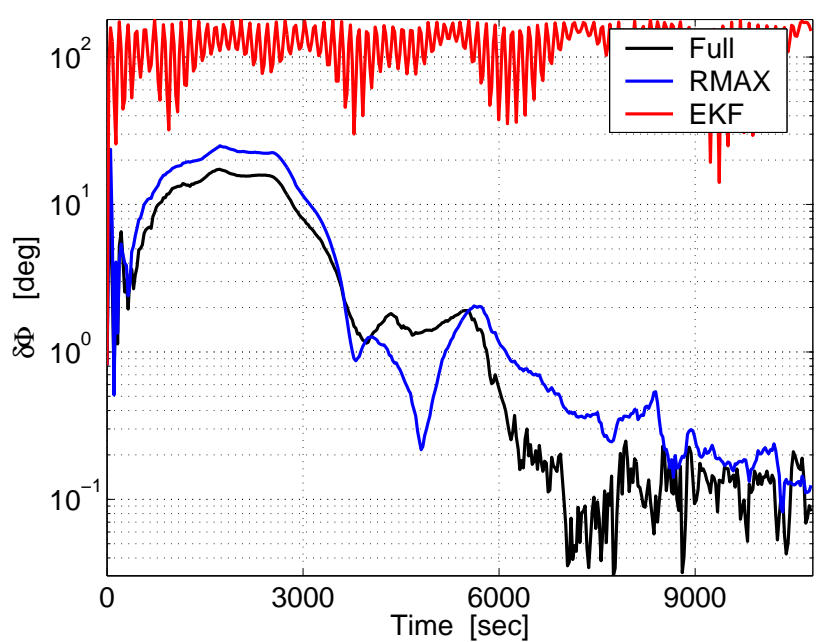

(b) Tumbling S/C: Angular estimation error.
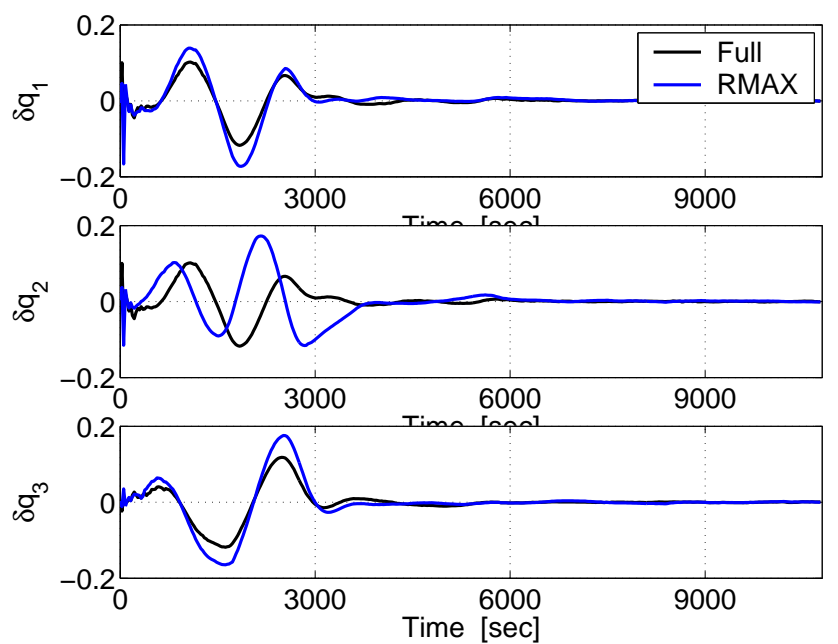

(d) Tumbling S/C: Quaternion error vector part.

Figure 15. Time histories of the estimation quaternion-error on a single run. For the spinning $\mathrm{S} / \mathrm{C}$ case,the three filters show equivalent performances. For the freely tumbling S/C case, the RMAX filter shows improved performances over the full filter, and the EKF fails to converge. 

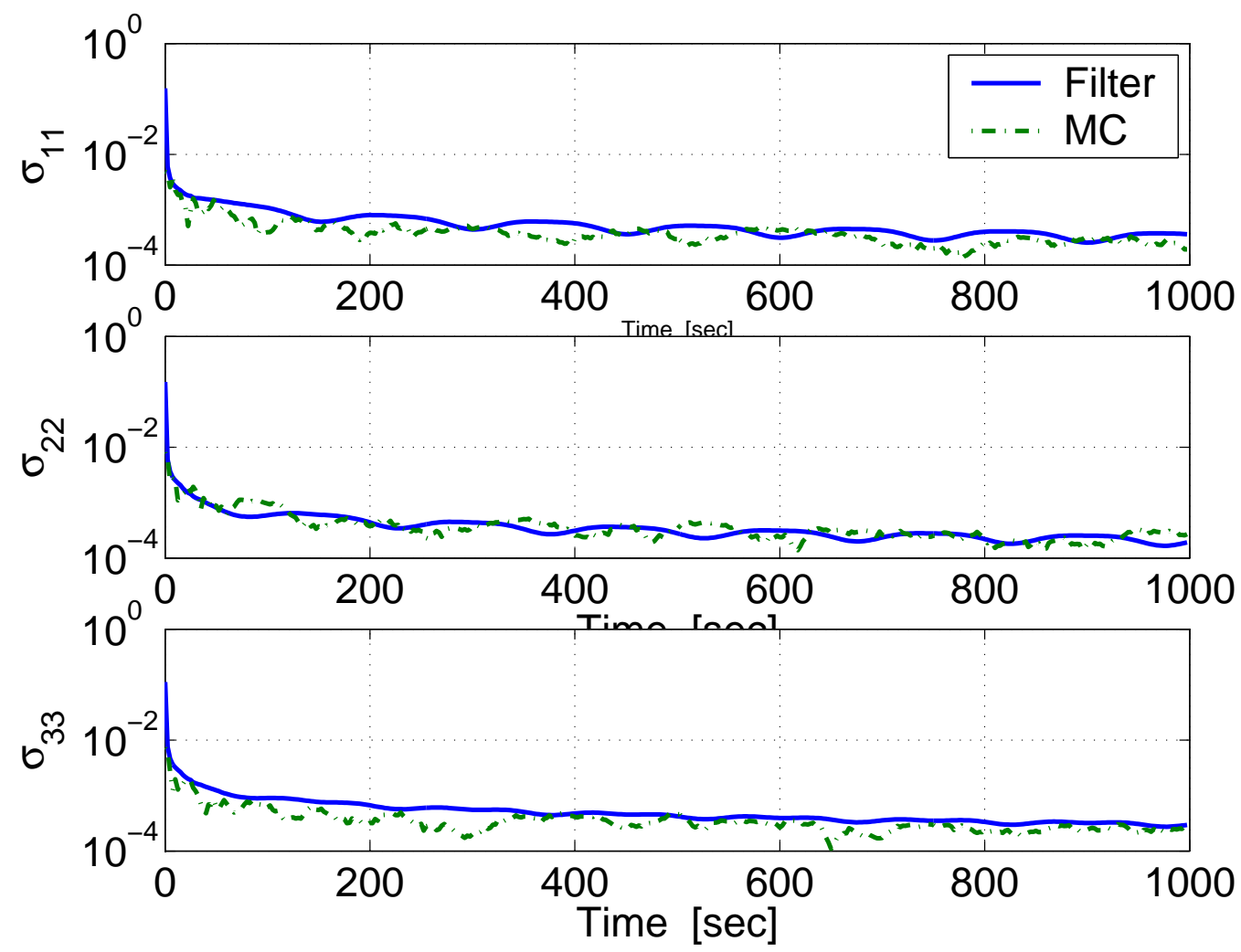

Figure 16. Time histories of the Monte-Carlo (MC) standard deviations for the quaternion-error vector part and comparison with the square-root of the diagonal elements in $P_{t / t}$. Without need for tuning, both plots are in agreement which denotes the statistical consistency of the maximal information rate quaternion filter. 60 MC-runs. 